EUROPEAN JOURNAL OF PURE AND APPLIED MATHEMATICS

Vol. 14, No. 4, 2021, 1324-1336

ISSN 1307-5543 - ejpam.com

Published by New York Business Global

\title{
On $k$-Cost Effective Domination Number in the Join of Graphs
}

\author{
Jesrael B. Palco ${ }^{1, *}$, Rolando N. Paluga ${ }^{2}$, Gina A. Malacas ${ }^{3}$ \\ 1 Department of Physical Sciences and Mathematics, College of Science and Environment, \\ Mindanao State University at Naawan, 9023, Naawan, Misamis Oriental, Philippines \\ 2 Department of Mathematics, College of Mathematics and Natural Sciences, \\ Caraga State University , 8600, Ampayon, Butuan City, Philippines \\ 3 Department of Mathematics and Statistics, College of Science and Mathematics, \\ Mindanao State University-Iligan Institute of Technology, 9200, Iligan City, Philippines
}

\begin{abstract}
In this paper, we characterized the $k$-cost effective domination in the join of graphs. Further, we investigate the $k$-cost effective domination, cost effective domination index, maximal cost effective domination in the join of graphs.
\end{abstract}

2020 Mathematics Subject Classifications: 05C69

Key Words and Phrases: $k$-cost effective set, $k$-cost effective domination index, maximal cost effective domination.

\section{Introduction}

Let $G=(V(G), E(G))$ be a connected simple graph and $v \in V(G)$. The neighborhood of $v$ in the set $N_{G}(v)=N(v)=\{u \in V(G): u v \in E(G)\}$. The degree of a vertex $v$ in a graph $G$, denoted by $\operatorname{deg}_{G}(v)$, is $|N(v)|$. A subset $S$ of $V(G)$ is a dominating set of $G$ if for every $v \in V(G) \backslash S$, there exists $u \in S$ such that $u v \in E(G)$. The domination number $\gamma(G)$ of $G$ is the minimum cardinality of a dominating set of $G$. A subset $S$ of $V(G)$ is an independent set of $G$ if $u v \notin E(G)$ for distinct pairs of vertices $u$ and $v$ in $S$. An independent dominating set in $G$ is an independent set in $G$ which is dominating in $G$. The minimum cardinality $\gamma_{i}(G)$ of an independent dominating set in $G$ is called independence domination number.

Let $k \geq 0$ be an integer. Consider a vertex $v$, its neighborhood set, $N(v)$ and the vertex-set of $G, V(G)$. A vertex $v \in S \subseteq V(G)$ is said to be $k$-cost effective if $|N(v) \cap(V(G) \backslash S)| \geq|N(v) \cap S|+k$. A dominating set $S$ is $k$-cost effective, if every vertex in $S$ is $k$-cost effective. The minimum cardinality of a $k$-cost effective dominating

${ }^{*}$ Corresponding author.

DOI: https://doi.org/10.29020/nybg.ejpam.v14i4.4117

Email addresses: jesrael.palco@msunaawan.edu.ph (J. B. Palco), rnpaluga@carsu.edu.ph (R. N. Paluga), gina.malacas@g.msuiit.edu.ph (G. A. Malacas) 
set of $G$ is the $k$-cost effective domination number $\gamma_{c e}^{k}(G)$ of $G$. In cases where there is no $k$-cost effective dominating set for $G$, the $k$-cost effective domination number of $G$ is infinity. The $k$-cost effective domination index of $G$, denoted by $\eta(G)$, is the maximum value of $k$ such that $k$-cost effective domination number is finite. That is,

$$
\eta(G)=\max \left\{k: \gamma_{c e}^{k}(G) \text { is finite. }\right\}
$$

The maximal cost effective domination number of $G$ is equal to $\gamma_{c e}^{\eta(G)}(G)$.

\section{Results}

Theorem 1. Let $G$ and $H$ be connected graphs, $k \geq \max \{|V(G)|,|V(H)|\}$, and $S \subseteq V(G+H)$. Then $S$ is a $k$-cost effective dominating set in $G+H$ if and only if one of the following holds:

(i) $S$ is $(k-|V(H)|)$-cost effective dominating set in $G$;

(ii) $S$ is $(k-|V(G)|)$-cost effective dominating set in $H$;

(iii) $V(G) \cap S$ is $\left(k-k_{1}\right)$-cost effective dominating set in $G$, where $k_{1}=|V(H)|-2|V(H) \cap S|$ and $V(H) \cap S$ is $\left(k-k_{2}\right)$-cost effective dominating set in $H$, where $k_{2}=|V(G)|-2|V(G) \cap S|$.

Proof: Let $k \geq \max \{|V(G)|,|V(H)|\}$, and $S \subseteq V(G+H)$. Suppose $S$ is a $k$-cost effective dominating set in $G+H$ and let $x \in S$. Then

$$
\left|N_{G+H}(x) \backslash S\right|-\left|N_{G+H}(x) \cap S\right| \geq k .
$$

Suppose $S \subseteq V(G)$. Then $S$ is a dominating set in $G$. Now,

$$
\begin{aligned}
\left|N_{G+H}(x) \backslash S\right|-\left|N_{G+H}(x) \cap S\right| & =|V(H)|+\left|N_{G}(x) \backslash S\right| \\
& -\left|N_{G}(x) \cap S\right| \\
& \geq k .
\end{aligned}
$$

This implies that,

$$
\left|N_{G}(x) \backslash S\right|-\left|N_{G}(x) \cap S\right| \geq k-|V(H)| .
$$

Hence, $S$ is $(k-|V(H)|)$-cost effective dominating set in $G$. Similarly, if $S \subseteq V(H)$, then $S$ is $(k-|V(G)|)$-cost effective dominating set in $H$.

Suppose that $S_{1}=V(G) \cap S \neq \varnothing$ and $S_{2}=V(H) \cap S \neq \varnothing$. Since $S$ is a $k$-cost effective dominating set in $G+H$,

$$
\left|N_{G+H}(x) \backslash S\right|-\left|N_{G+H}(x) \cap S\right| \geq k .
$$

Let $x \in S_{1} \subseteq S$. Then

$$
\left|N_{G+H}(x) \backslash S\right|-\left|N_{G+H}(x) \cap S\right|=\left|N_{G}(x) \backslash S_{1}\right|+\left|V(H) \backslash S_{2}\right|-\left|N_{G}(x) \cap S_{1}\right|-\left|S_{2}\right|
$$




$$
\begin{aligned}
& =\left|N_{G}(x) \backslash S_{1}\right|+|V(H)|-\left|S_{2}\right|-\left|N_{G}(x) \cap S_{1}\right|-\left|S_{2}\right| \\
& =\left|N_{G}(x) \backslash S_{1}\right|-\left|N_{G}(x) \cap S_{1}\right|+|V(H)|-2\left|S_{2}\right| .
\end{aligned}
$$

This implies that,

$$
\begin{aligned}
\left|N_{G}(x) \backslash S_{1}\right|-\left|N_{G}(x) \cap S_{1}\right| & \geq k-|V(H)|+2|V(H) \cap S| \\
& =k-(|V(H)|-2|V(H) \cap S|) \\
& =k-k_{1},
\end{aligned}
$$

where $k_{1}=|V(H)|-2|V(H) \cap S|$. Thus, $S_{1}=V(G) \cap S$ is $\left(k-k_{1}\right)$-cost effective dominating set in $G$. Similarly, $S_{2}=V(H) \cap S$ is $\left(k-k_{2}\right)$-cost effective dominating set in $H$.

Conversely, suppose that $S$ satisfies Property $(i)$. Then $S$ is a dominating set in $G+H$ and

$$
\left|N_{G}(x) \backslash S\right|-\left|N_{G}(x) \cap S\right| \geq k-|V(H)|, \forall x \in S .
$$

Now,

$$
\begin{aligned}
\left|N_{G+H}(x) \backslash S\right|-\left|N_{G+H}(x) \cap S\right| & =|V(H)|+\left|N_{G}(x) \backslash S\right| \\
& -\left|N_{G}(x) \cap S\right| \\
& \geq|V(H)|+k-|V(H)| \\
& =k
\end{aligned}
$$

for all $x \in S$. Since $x$ is arbitrary, $S$ is a $k$-cost effective dominating set in $G+H$. Similarly, if $S$ satisfies Property (ii), then $S$ is a $k$-cost effective dominating set in $G+H$. Suppose $S$ satisfies Property (iii) and $x \in V(G) \cap S$. Then

$$
\left|N_{G}(x) \backslash S\right|-\left|N_{G}(x) \cap S\right| \geq k-k_{1},
$$

where $k_{1}=|V(H)|-2|V(H) \cap S|$. Now,

$$
\begin{aligned}
\left|N_{G+H}(x) \backslash S\right|-\left|N_{G+H}(x) \cap S\right| & =\left|N_{G}(x) \backslash S\right|+|V(H) \backslash S|-\left|N_{G}(x) \cap S\right|+|V(H) \cap S| \\
& =\left|N_{G}(x) \backslash S\right|-\left|N_{G}(x) \cap S\right|+|V(H) \backslash S|-|V(H) \cap S| \\
& =\left|N_{G}(x) \backslash S\right|-\left|N_{G}(x) \cap S\right|+|V(H)|-2|V(H) \cap S| \\
& \geq k-k_{1}+k_{1} \\
& =k .
\end{aligned}
$$

Similarly, for each $x \in V(H) \cap S,\left|N_{G+H}(x) \backslash S\right|-\left|N_{G+H}(x) \cap S\right| \geq k$. Therefore, $S$ is a $k$-cost effective dominating set in $G+H$.

Corollary 1. Let $G$ and $H$ be connected graphs, $k \geq \max \{|V(G)|,|V(H)|\}$. If $S$ is a $k$-cost effective dominating set in $G+H$, then one of the following holds:

(i) $S \subseteq V(G)$ and $k \leq \eta(G)+|V(H)|$;

(ii) $S \subseteq V(H)$ and $k \leq \eta(H)+|V(G)|$; 
(iii) $k \leq \min \{\eta(G)+|V(H)|-2|V(H) \cap S|, \eta(H)+|V(G)|-2|V(G) \cap S|\}$.

Theorem 2. Let $G$ and $H$ be connected graphs such that $\gamma(G)=1$ or $\gamma(H)=1$ and $0 \leq k \leq|V(H)|+|V(G)|-1$. Then $S \subseteq V(G+H)$ is a $\gamma_{c e}^{k}$-set in $G+H$ if and only if $S$ is a $\gamma$-set in $G$ or $S$ is a $\gamma$-set in $H$.

Corollary 2. Let $G$ and $H$ be connected graphs such that $\gamma(G)=1$ or $\gamma(H)=1$. Then

$$
\gamma_{c e}^{k}(G+H)= \begin{cases}1, & \text { if } 0 \leq k \leq|V(H)|+|V(G)|-1 \\ \infty, & \text { if } k>|V(H)|+|V(G)|-1 .\end{cases}
$$

Corollary 3. Let $G$ and $H$ be connected graphs such that $\gamma(G)=1$ or $\gamma(H)=1$. Then $\eta(G+H)=|V(H)|+|V(G)|-1$ and $\gamma_{c e}^{\eta(G+H)}(G+H)=1$.

In the succeeding theorems, $\gamma(G) \geq 2$ and $\gamma(H) \geq 2$ and assume that $\Delta(G)+|V(H)| \leq \Delta(H)+|V(G)|$.

Theorem 3. Let $G$ and $H$ be connected graphs such that $\min \{\gamma(G), \gamma(H)\} \geq 2$ and $0 \leq k \leq \Delta(G)+|V(H)|-2$. Then $S$ is a $\gamma_{c e}^{k}$-set in $G+H$ if and only if $|S|=2$ and one of the following holds:

(i) $|V(G) \cap S|=1$ and $|V(H) \cap S|=1$;

(ii) $S$ is a $\gamma$-set in $G$ such that $k-|V(H)|+2 \leq \delta(S: G)$;

(iii) $S$ is a $\gamma$-set in $H$ such that $k-|V(G)|+2 \leq \delta(S: H)$.

Proof: Suppose that $A=\{a, b\}$ such that $\operatorname{deg}_{G}(a)=\Delta(G)$ and $\operatorname{deg}_{H}(b)=\Delta(H)$. Clearly, $A$ is a dominating set in $G+H$. Moreover,

$$
\begin{aligned}
\left|N_{G+H}(a) \backslash A\right|-\left|N_{G+H}(a) \cap A\right| & =\operatorname{deg}_{G}(a)+|V(H)| \\
& =\Delta(G)+|V(H)| \\
& >\Delta(G)+|V(H)|-2 \\
& \geq k .
\end{aligned}
$$

and

$$
\begin{aligned}
\left|N_{G+H}(b) \backslash A\right|-\left|N_{G+H}(b) \cap A\right| & =\operatorname{deg}_{H}(b)+|V(G)| \\
& =\Delta(H)+|V(G)| \\
& \geq \Delta(G)+|V(H)| \\
& >\Delta(G)+|V(H)|-2 \\
& \geq k .
\end{aligned}
$$

Thus, $A$ is a $k$-cost effective dominating set in $G+H$. Accordingly, $\gamma_{c e}^{k}(G+H)=|S| \leq 2$. Suppose that $|S|=1$. Then $\gamma(G)=1$ or $\gamma(H)=1$, which is 
a contradiction to that fact that $\min \{\gamma(G), \gamma(H)\} \geq 2$. Therefore, $\gamma_{c e}^{k}(G+H)=2$. Since $S$ is a $\gamma_{c e}^{k}$-set in $G+H,|S|=2$.

Clearly, $|V(G) \cap S|=1$ and $|V(H) \cap S|=1$. Thus, Property $(i)$ holds.

Suppose that $S \subseteq V(G)$. Since $S$ is a dominating set in $G+H, S$ is a dominating set in $G$. Now, $\gamma(G) \geq 2$, so $S$ is a minimum dominating set in $G$, that is, $S$ is a $\gamma$-set in $G$. Let $S=\left\{a_{1}, a_{2}\right\} \subseteq V(G)$. Suppose $a_{1}$ and $a_{2}$ are adjacent in $S$. Then

$$
\begin{aligned}
\left|N_{G+H}\left(a_{i}\right) \backslash S\right|-\left|N_{G+H}\left(a_{i}\right) \cap S\right| & =\left(|V(H)|+\operatorname{deg}_{G}\left(a_{i}\right)-1\right)-1 \\
& =|V(H)|+\operatorname{deg}_{G}\left(a_{i}\right)-2 \\
& \geq|V(H)|+\delta(S: G)-2 \\
& \geq k, i=1,2 .
\end{aligned}
$$

Thus, $k-|V(H)|+2 \leq \delta(S: G)$. Suppose $a_{1}$ and $a_{2}$ are not adjacent in $S$. Then

$$
\begin{aligned}
\left|N_{G+H}\left(a_{i}\right) \backslash S\right|-\left|N_{G+H}\left(a_{i}\right) \cap S\right| & =|V(H)|+\operatorname{deg}_{G}\left(a_{i}\right) \\
& >|V(H)|+\operatorname{deg}_{G}\left(a_{i}\right)-2 \\
& \geq|V(H)|+\delta(S: G)-2 \\
& =k, \quad i=1,2 .
\end{aligned}
$$

Thus, $k-|V(H)|+2 \leq \delta(S: G)$. Similarly, $k-|V(G)|+2 \leq \delta(S: H)$.

Conversely, suppose that $S$ satisfies Property $(i)$. Then $S$ is a $\gamma$-set in $G+H$. Moreover,

$$
\begin{aligned}
\left|N_{G+H}(a) \backslash S\right|-\left|N_{G+H}(a) \cap S\right| & =|V(H)|-1+\operatorname{deg}_{G}(a)-1 \\
& =|V(H)|+\Delta(G)-2 \\
& \geq k .
\end{aligned}
$$

and

$$
\begin{aligned}
\left|N_{G+H}(b) \backslash S\right|-\left|N_{G+H}(b) \cap S\right| & =|V(G)|-1+\operatorname{deg}_{H}(b)-1 \\
& =|V(G)|+\Delta(H)-2 \\
& =|V(H)|+\Delta(G)-2 \\
& \geq k
\end{aligned}
$$

Thus, $S$ is a $k$-cost effective dominating set in $G+H$. Hence, $S$ is a $\gamma_{c e}^{k}$-set in $G+H$. Suppose that $S$ satisfies Property (ii). Then $S$ is a $\gamma$-set in $G+H$. Suppose $a_{1}$ and $a_{2}$ are adjacent in $S$. Then

$$
\begin{aligned}
\left|N_{G+H}\left(a_{i}\right) \backslash S\right|-\left|N_{G+H}\left(a_{i}\right) \cap S\right| & =\left(|V(H)|+\operatorname{deg}_{G}\left(a_{i}\right)-1\right)-1 \\
& =|V(H)|+\operatorname{deg}_{G}\left(a_{i}\right)-2 \\
& \geq|V(H)|+\delta(S: G)-2 \\
& \geq k .
\end{aligned}
$$


Suppose $a_{1}$ and $a_{2}$ are not adjacent in $S$. Then

$$
\begin{aligned}
\left|N_{G+H}\left(a_{i}\right) \backslash S\right|-\left|N_{G+H}\left(a_{i}\right) \cap S\right| & =|V(H)|+\operatorname{deg}_{G}\left(a_{i}\right) \\
& >|V(H)|+\operatorname{deg}_{G}\left(a_{i}\right)-2 \\
& \geq|V(H)|+\delta(S: G)-2 \\
& =k .
\end{aligned}
$$

Thus, $S$ is a $k$-cost effective dominating set in $G+H$. Suppose that a singleton set is a dominating set in $G+H$. Then $\gamma(G)=1$ or $\gamma(H)=1$, which a contradiction to the fact that $\min \{\gamma(G), \gamma(H)\} \geq 2$. Hence, $S$ is a $\gamma_{c e}^{k}$-set in $G+H$. Similarly, if $S$ satisfies Property (iii), then $S$ is a $\gamma_{c e}^{k}$-set in $G+H$.

Therefore, $S$ is a $\gamma_{c e}^{k}$-set in $G+H$.

Theorem 4. Let $G$ and $H$ be connected graphs such that $\min \{\gamma(G), \gamma(H)\} \geq 2$ and $k=\Delta(G)+|V(H)|-1$. Then $S$ is a $k$-cost effective dominating set in $G+H$ if and only if one of the following holds:

(i) $S$ is an independent dominating set in $G$ such that $\delta(S: G) \geq \Delta(G)-1$;

(ii) $S$ is a dominating set in $H$ such that $0 \leq r_{H}(a)+2\left|N_{H}(a) \cap S\right|-t \leq 1$, where $r_{H}(a)=\Delta(H)-\operatorname{deg}_{H}(a)$ and $t=\Delta(H)+|V(G)|-\Delta(G)-|V(H)|$, and $\operatorname{deg}_{H}(a)+|V(G)|-2\left|N_{H}(a) \cap S\right|=\Delta(G)+|V(H)|-1$.

Proof: Suppose that $S$ is a $k$-cost effective dominating set in $G+H$. Consider the following cases:

Case 1: $V(G) \cap S \neq \varnothing$ and $V(H) \cap S \neq \varnothing$.

Let $a \in V(G) \cap S$. Then

$$
\begin{aligned}
\left|N_{G+H}(a) \backslash S\right|-\left|N_{G+H}(a) \cap S\right| & \leq \Delta(G)-1+|V(H)|-1 \\
& <\Delta(G)+|V(H)|-1 \\
& =k,
\end{aligned}
$$

a contradiction. Thus, this case is not possible.

Case 2: $S \subseteq V(G)$.

Suppose $S$ is not an independent dominating set G. Let $a \in S$. Then there exists $a^{\prime} \in S$ such that $d_{G}\left(a, a^{\prime}\right)=1$. Now

$$
\begin{aligned}
\left|N_{G+H}(a) \backslash S\right|-\left|N_{G+H}(a) \cap S\right| & \leq \Delta(G)-1+|V(H)|-1 \\
& <\Delta(G)+|V(H)|-1 \\
& =k,
\end{aligned}
$$

a contradiction. Thus, in this case $S$ is an independent dominating set in $G$. Let $r_{G}(a)=\Delta(H)-\operatorname{deg}_{G}(a)$. Now, $S$ is a $k$-cost effective dominating set in $G+H$, so

$$
\left|N_{G+H}(a) \backslash S\right|-\left|N_{G+H}(a) \cap S\right|=\operatorname{deg}_{G}(a)+|V(H)|
$$




$$
\begin{aligned}
& =\Delta(G)-r_{G}(a)+|V(H)| \\
& \geq \Delta(G)+|V(H)|-1 .
\end{aligned}
$$

Thus, $r_{G}(a) \leq 1$ and $\operatorname{deg}_{G}(a) \geq \Delta(G)-1$ for all $a \in S$. Hence, $\delta(S: G) \geq \Delta(G)-1$.

Case 3: $S \subseteq V(H)$.

Since $S$ is a $k$-cost effective dominating set in $G+H, S$ is a dominating set in $H$. Let $a \in S$ and $r_{H}(a)=\Delta(H)-\operatorname{deg}_{H}(a)$, and $t=\Delta(H)+|V(G)|-\Delta(G)-|V(H)|$. Then

$$
\begin{aligned}
\left|N_{G+H}(a) \backslash S\right|-\left|N_{G+H}(a) \cap S\right| & =\operatorname{deg}_{H}(a)-\left|N_{H}(a) \cap S\right|+|V(G)|-\left|N_{H}(a) \cap S\right| \\
& =\Delta(H)-r_{H}(a)-2\left|N_{H}(a) \cap S\right|+|V(G)| \\
& =\Delta(G)+|V(H)|+t-r_{H}(a)-2\left|N_{H}(a) \cap S\right| \\
& =\Delta(G)+|V(H)|-\left(r_{H}(a)+2\left|N_{H}(a) \cap S\right|-t\right) .
\end{aligned}
$$

Thus, $0 \leq r_{H}(a)+2\left|N_{H}(a) \cap S\right|-t \leq 1$. Hence, $\operatorname{deg}_{H}(a)+|V(G)|-2\left|N_{H}(a) \cap S\right|=$ $\Delta(G)+|V(H)|-1$.

Conversely, suppose that $S$ satisfies Property $(i)$. Then $S$ is a dominating set in $G+H$. Let $a \in S$. Then

$$
\begin{aligned}
\left|N_{G+H}(a) \backslash S\right|-\left|N_{G+H}(a) \cap S\right| & =\operatorname{deg}_{G}(a)+|V(H)| \\
& =\Delta(G)-1+|V(H)| \\
& =k .
\end{aligned}
$$

Hence, $S$ is a $k$-cost effective dominating set in $G+H$.

Suppose that $S$ satisfies Property (ii). Then $S$ is a dominating set in $G+H$. Now,

$$
\begin{aligned}
\left|N_{G+H}(a) \backslash S\right|-\left|N_{G+H}(a) \cap S\right| & =\operatorname{deg}_{H}(a)-\left|N_{H}(a) \cap S\right|+|V(G)|-\left|N_{H}(a) \cap S\right| \\
& =\Delta(H)-r_{H}(a)-2\left|N_{H}(a) \cap S\right|+|V(G)| \\
& =\Delta(G)+|V(H)|+t-r_{H}(a)-2\left|N_{H}(a) \cap S\right| \\
& =\Delta(G)+|V(H)|-\left(r_{H}(a)+2\left|N_{H}(a) \cap S\right|-t\right) .
\end{aligned}
$$

If $r_{H}(a)+2\left|N_{H}(a) \cap S\right|-t=0$, then

$$
\begin{aligned}
\left|N_{G+H}(a) \backslash S\right|-\left|N_{G+H}(a) \cap S\right| & =\operatorname{deg}_{H}(a)-\left|N_{H}(a) \cap S\right|+|V(G)|-\left|N_{H}(a) \cap S\right| \\
& =\Delta(H)-r_{H}(a)-2\left|N_{H}(a) \cap S\right|+|V(G)| \\
& =\Delta(G)+|V(H)|+t-r_{H}(a)-2\left|N_{H}(a) \cap S\right| \\
& =\Delta(G)+|V(H)|-\left(r_{H}(a)+2\left|N_{H}(a) \cap S\right|-t\right. \\
& =\Delta(G)+|V(H)| \\
& >\Delta(G)+|V(H)|-1 \\
& =k .
\end{aligned}
$$

Hence, $S$ is a $k$-cost effective dominating set in $G+H$. If

$$
r_{H}(a)+2\left|N_{H}(a) \cap S\right|-t=1
$$


then

$$
\begin{aligned}
\left|N_{G+H}(a) \backslash S\right|-\left|N_{G+H}(a) \cap S\right| & =\operatorname{deg}_{H}(a)-\left|N_{H}(a) \cap S\right|+|V(G)|-\left|N_{H}(a) \cap S\right| \\
& =\Delta(H)-r_{H}(a)-2\left|N_{H}(a) \cap S\right|+|V(G)| \\
& =\Delta(G)+|V(H)|+t-r_{H}(a)-2\left|N_{H}(a) \cap S\right| \\
& =\Delta(G)+|V(H)|-\left(r_{H}(a)+2\left|N_{H}(a) \cap S\right|-t\right. \\
& =\Delta(G)+|V(H)|-1 \\
& =k .
\end{aligned}
$$

Hence, $S$ is a $k$-cost effective dominating set in $G+H$.

Therefore, $S$ is a $k$-cost effective dominating set in $G+H$.

Theorem 5. Let $G$ and $H$ be connected graphs such that $\min \{\gamma(G), \gamma(H)\} \geq 2$ and $k=\Delta(G)+|V(H)|$. Then $S$ is a $k$-cost effective dominating set in $G+H$ if and only if one of the following holds:

(i) $S$ is an independent dominating set in $G$ such that $\delta(S: G)=\Delta(G)$;

(ii) $S$ is a dominating set in $H$ such that $\operatorname{deg}_{H}(a)+|V(G)|=2\left|N_{H}(a) \cap S\right|+\Delta(G)+|V(H)|$ and $r_{H}(a)+2\left|N_{H}(a) \cap S\right|-t=0$, where $r_{H}(a)=\Delta(H)-\operatorname{deg}_{H}(a)$, $t=\Delta(H)+|V(G)|-\Delta(G)-|V(H)|$.

Proof: Suppose that $S$ is a $k$-cost effective dominating set in $G+H$. Consider the following cases:

Case 1: $V(G) \cap S \neq \varnothing$ and $V(H) \cap S \neq \varnothing$.

Let $a \in V(G) \cap S$. Then

$$
\begin{aligned}
\left|N_{G+H}(a) \backslash S\right|-\left|N_{G+H}(a) \cap S\right| & \leq \Delta(G)-1+|V(H)|-1 \\
& <\Delta(G)+|V(H)| \\
& =k,
\end{aligned}
$$

a contradiction. Thus, this case is not possible.

Case 2: $S \subseteq V(G)$.

Suppose $S$ is not an independent dominating set G. Let $a \in S$. Then there exists $a^{\prime} \in S$ such that $d_{G}\left(a, a^{\prime}\right)=1$. Now

$$
\begin{aligned}
\left|N_{G+H}(a) \backslash S\right|-\left|N_{G+H}(a) \cap S\right| & \leq \Delta(G)-1+|V(H)|-1 \\
& <\Delta(G)+|V(H)| \\
& =k,
\end{aligned}
$$

a contradiction. Thus, in this case $S$ is an independent dominating set in $G$. Now,

$$
\begin{aligned}
\left|N_{G+H}(a) \backslash S\right|-\left|N_{G+H}(a) \cap S\right| & =\operatorname{deg}_{G}(a)+|V(H)| \\
& =\Delta(G)+|V(H)|
\end{aligned}
$$




$$
=k,
$$

Thus, $\operatorname{deg}_{G}(a)=\Delta(G) \forall a \in S$. Hence, $\delta(S: G)=\Delta(G)$.

Case 3: $S \subseteq V(H)$.

Since $S$ is a $k$-cost effective dominating set in $G+H, S$ is a dominating set in $H$. Let $a \in S$ and $r_{H}(a)=\Delta(H)-\operatorname{deg}_{H}(a)$, and $t=\Delta(H)+|V(G)|-\Delta(G)-|V(H)|$. Then

$$
\begin{aligned}
\left|N_{G+H}(a) \backslash S\right|-\left|N_{G+H}(a) \cap S\right| & =\operatorname{deg}_{H}(a)-\left|N_{H}(a) \cap S\right|+|V(G)|-\left|N_{H}(a) \cap S\right| \\
& =\Delta(H)-r_{H}(a)-2\left|N_{H}(a) \cap S\right|+|V(G)| \\
& =\Delta(G)+|V(H)|+t-r_{H}(a)-2\left|N_{H}(a) \cap S\right| \\
& =\Delta(G)+|V(H)|-\left(r_{H}(a)+2\left|N_{H}(a) \cap S\right|-t\right) .
\end{aligned}
$$

Thus, $r_{H}(a)+2\left|N_{H}(a) \cap S\right|-t=0$. Hence, $\operatorname{deg}_{H}(a)+|V(G)|=2\left|N_{H}(a) \cap S\right|+\Delta(G)+$ $|V(H)|$.

Conversely, suppose that $S$ satisfies Property $(i)$. Then $S$ is a dominating set in $G+H$. Let $a \in S$. Then

$$
\begin{aligned}
\left|N_{G+H}(a) \backslash S\right|-\left|N_{G+H}(a) \cap S\right| & =\operatorname{deg}_{G}(a)+|V(H)| \\
& =\delta(S: G)+|V(H)| \\
& =\Delta(G)+|V(H)| \\
& =k .
\end{aligned}
$$

Hence, $S$ is a $k$-cost effective dominating set in $G+H$.

Suppose that $S$ satisfies Property ( $i i)$. Then $S$ is a dominating set in $G+H$. Now,

$$
\begin{aligned}
\left|N_{G+H}(a) \backslash S\right|-\left|N_{G+H}(a) \cap S\right|= & \operatorname{deg}_{H}(a)-\left|N_{H}(a) \cap S\right|+|V(G)|-\left|N_{H}(a) \cap S\right| \\
= & \Delta(H)-r_{H}(a)-2\left|N_{H}(a) \cap S\right|+|V(G)| \\
= & \Delta(G)+|V(H)|+t-r_{H}(a)-2\left|N_{H}(a) \cap S\right| \\
= & \Delta(G)+|V(H)|+\Delta(H)+|V(G)|-\Delta(G) \\
& \quad-|V(H)|-\Delta(H)+\operatorname{deg}_{H}(a)-2\left|N_{H}(a) \cap S\right| \\
= & |V(G)|+\operatorname{deg}_{H}(a)-2\left|N_{H}(a) \cap S\right| \\
= & \Delta(G)+|V(H)| \\
= & k .
\end{aligned}
$$

Thus, $S$ is a $k$-cost effective dominating set in $G+H$.

Therefore, $S$ is a $k$-cost effective dominating set in $G+H$.

Theorem 6. Let $G$ and $H$ be connected graphs such that $\min \{\gamma(G), \gamma(H)\} \geq 2$ and $\Delta(G)+|V(H)|+1 \leq k \leq \Delta(H)+|V(G)|$. Then $S$ is a $k$-cost effective dominating set in $G+H$ if and only if $S$ is a dominating set in $H$ such that $t-r_{H}(a)-2\left|N_{H}(a) \cap S\right| \geq p$, where $1 \leq p \leq t$ and $t=\Delta(H)+|V(G)|-\Delta(G)-|V(H)|$, and $r_{H}(a)=\Delta(H)-\operatorname{deg}_{H}(a)$ and $\operatorname{deg}_{H}(a)+|V(G)| \geq p+2\left|N_{H}(a) \cap S\right|+\Delta(G)+|V(H)|$. 
Proof: Suppose that $S$ is a $k$-cost effective dominating set in $G+H$. Consider the following cases:

Case 1: $V(G) \cap S \neq \varnothing$ and $V(H) \cap S \neq \varnothing$.

Let $a \in V(G) \cap S$. Then

$$
\begin{aligned}
\left|N_{G+H}(a) \backslash S\right|-\left|N_{G+H}(a) \cap S\right| & \leq \Delta(G)-1+|V(H)|-1 \\
& <\Delta(G)+|V(H)|+1 \\
& \leq k,
\end{aligned}
$$

a contradiction. Thus, in this case is not possible.

Case 2: $S \subseteq V(G)$.

Suppose $S$ is not an independent dominating set G. Let $a \in S$. Then there exists $a^{\prime} \in S$ such that $d_{G}\left(a, a^{\prime}\right)=1$. Now

$$
\begin{aligned}
\left|N_{G+H}(a) \backslash S\right|-\left|N_{G+H}(a) \cap S\right| & \leq \Delta(G)-1+|V(H)|-1 \\
& <\Delta(G)+|V(H)|+1 \\
& \leq k,
\end{aligned}
$$

a contradiction. Thus, in this case $S$ is an independent dominating set in $G$. Thus,

$$
\begin{aligned}
\left|N_{G+H}(a) \backslash S\right|-\left|N_{G+H}(a) \cap S\right| & =\operatorname{deg}_{G}(a)+|V(H)| \\
& =\Delta(G)-r_{G}(a)+|V(H)| \\
& \leq \Delta(G)+|V(H)|+1 \\
& \geq k,
\end{aligned}
$$

a contradiction. Thus, in this case is not possible.

Case 3: $S \subseteq V(H)$.

Since $S$ is a $k$-cost effective dominating set in $G+H, S$ is a dominating set in $H$. Let $a \in S, r_{H}(a)=\Delta(H)-\operatorname{deg}_{H}(a)$ and $1 \leq p \leq t$, where $t=\Delta(H)+|V(G)|-\Delta(G)-|V(H)|$. Then

$$
\begin{aligned}
\left|N_{G+H}(a) \backslash S\right|-\left|N_{G+H}(a) \cap S\right| & =\operatorname{deg}_{H}(a)-\left|N_{H}(a) \cap S\right|+|V(G)|-\left|N_{H}(a) \cap S\right| \\
& =\Delta(H)-r_{H}(a)-2\left|N_{H}(a) \cap S\right|+|V(G)| \\
& =\Delta(G)+|V(H)|+t-r_{H}(a)-2\left|N_{H}(a) \cap S\right| .
\end{aligned}
$$

Thus, $t-r_{H}(a)-2\left|N_{H}(a) \cap S\right| \geq p$. Hence, $\operatorname{deg}_{H}(a)+|V(G)| \geq p+2\left|N_{H}(a) \cap S\right|+\Delta(G)+$ $|V(H)|$.

Conversely, suppose that $S$ is a dominating set in $H$ such that $t-r_{H}(a)-2\left|N_{H}(a) \cap S\right| \geq p$, where $1 \leq p \leq t$ and $t=\Delta(H)+|V(G)|-\Delta(G)-|V(H)|$, and $r_{H}(a)=\Delta(H)-\operatorname{deg}_{H}(a)$ and $\operatorname{deg}_{H}(a)+|V(G)| \geq p+2\left|N_{H}(a) \cap S\right|+\Delta(G)+|V(H)|$. Then

$$
\left|N_{G+H}(a) \backslash S\right|-\left|N_{G+H}(a) \cap S\right|=\operatorname{deg}_{H}(a)-\left|N_{H}(a) \cap S\right|+|V(G)|-\left|N_{H}(a) \cap S\right|
$$




$$
\begin{aligned}
& =\Delta(H)-r_{H}(a)-2\left|N_{H}(a) \cap S\right|+|V(G)| \\
& =\Delta(G)+|V(H)| \\
& =k .
\end{aligned}
$$

Hence, $S$ is a $k$-cost effective dominating set in $G+H$.

Theorem 7. Let $G$ and $H$ be connected graphs such that $\min \{\gamma(G), \gamma(H)\} \geq 2$ and $k \geq \Delta(H)+|V(G)|+1$. Then $\gamma_{c e}^{k}(G+H)=\infty$.

Proof: Let $k \geq \Delta(H)+|V(G)|+1$. Suppose that there exists a $k$-cost effective dominating set $S$ in $G+H$. Consider the following cases:

Case 1: $V(G) \cap S \neq \varnothing$ and $V(H) \cap S \neq \varnothing$.

Let $a \in V(H) \cap S$. Then

$$
\begin{aligned}
\left|N_{G+H}(a) \backslash S\right|-\left|N_{G+H}(a) \cap S\right| & \leq \Delta(H)-1+|V(G)|-1 \\
& <\Delta(H)+|V(G)|+1 \\
& =k,
\end{aligned}
$$

a contradiction.

Case 2: $S \subseteq V(G)$.

Suppose $S$ is not an independent dominating set G. Let $a \in S$. Then there exists $a^{\prime} \in S$ such that $d_{G}\left(a, a^{\prime}\right)=1$. Now

$$
\begin{aligned}
\left|N_{G+H}(a) \backslash S\right|-\left|N_{G+H}(a) \cap S\right| & \leq \Delta(G)-1+|V(H)|-1 \\
& \leq \Delta(H)-1+|V(G)|-1 \\
& <\Delta(H)+|V(G)|+1 \\
& =k,
\end{aligned}
$$

a contradiction. Thus, in this case $S$ is an independent dominating set in $G$. Thus,

$$
\begin{aligned}
\left|N_{G+H}(a) \backslash S\right|-\left|N_{G+H}(a) \cap S\right| & =\operatorname{deg}_{G}(a)+|V(H)| \\
& =\Delta(G)-r_{G}(a)+|V(H)| \\
& =\Delta(H)-r_{G}(a)+|V(G)| \\
& <\Delta(H)+|V(G)|+1 \\
& =k,
\end{aligned}
$$

a contradiction.

Case 3: $S \subseteq V(H)$. . Let $a \in S$. Then

$$
\begin{aligned}
\left|N_{G+H}(a) \backslash S\right|-\left|N_{G+H}(a) \cap S\right| & =\operatorname{deg}_{H}(a)-\left|N_{H}(a) \cap S\right|+|V(G)|-\left|N_{H}(a) \cap S\right| \\
& =\Delta(H)-r_{H}(a)-2\left|N_{H}(a) \cap S\right|+|V(G)| \\
& =\Delta(G)+|V(H)|+t-r_{H}(a)-2\left|N_{H}(a) \cap S\right| \\
& =\Delta(G)+|V(H)|-\left(r_{H}(a)+2\left|N_{H}(a) \cap S\right|-t\right)
\end{aligned}
$$


REFERENCES

$$
\begin{aligned}
& =\Delta(H)+|V(G)|-\left(r_{H}(a)+2\left|N_{H}(a) \cap S\right|-t\right) \\
& <\Delta(H)+|V(G)|+1 \\
& =k
\end{aligned}
$$

a contradiction. Hence, $\gamma_{c e}^{k}(G+H)=\infty$.

The next result follows from Theorem 3, Theorem 4, Theorem 5, Theorem 6 and Theorem 7 .

Corollary 4. Let $G$ and $H$ be connected graphs such that $\gamma(G) \geq 2, \gamma(H) \geq 2$ and $|V(H)|+\Delta(G) \leq|V(G)|+\Delta(H)$. Then

$$
\gamma_{c e}^{k}(G+H)=\left\{\begin{array}{ll}
2, & \text { if } 0 \leq k \leq|V(G)|+\Delta(H)-2 \\
\min \left\{\gamma_{i}^{*}(G), \gamma^{*}(H)\right\}, & \text { if }|V(G)|+\Delta(H)-1 \leq k \leq \Delta(G)+|V(H)| \\
\gamma(H), & \text { if }|V(H)|+\Delta(G)+1 \leq k \leq|V(G)|+\Delta(H) \\
\infty & \text { if } k \geq|V(G)|+\Delta(H)+1
\end{array},\right.
$$

where

$\gamma_{i}^{*}(G)=\min \left\{|S|: S\right.$ is a $\gamma_{i}$-set in $G$ and $\left.\delta(S: G) \geq \Delta(G)-1\right\}$,

$\gamma^{*}(H)=\min \left\{|S|: S\right.$ is a $\gamma$-set in $G$ and $0 \leq \Delta(G)+|V(H)|-|V(G)|-\operatorname{deg}_{H}(a)+$ $\left.2\left|N_{H}(a) \cap S\right| \leq 1\right\}$, and

$\gamma(H)=\min \left\{|S|: S\right.$ is a $\gamma$-set in $G$ and $\left.\operatorname{deg}_{H}(a)+|V(G)|-|V(H)|-2\left|N_{H}(a) \cap S\right| \geq p\right\}$

Corollary 5. Let $G$ and $H$ be connected graphs such that $\gamma(G) \geq 2, \gamma(H) \geq 2$ and $|V(H)|+\Delta(G) \leq|V(G)|+\Delta(H)$. Then $\eta(G+H)=|V(G)|+\Delta(H)$ and $\gamma_{c e}^{\eta(G+H)}(G+H)=\gamma(H)$.

\section{Acknowledgements}

The authors thank the peer reviewers of the paper and readers of European Journal of Pure and Applied Mathematics, for making the journal successful.

\section{References}

[1] M. Chellali, T. W. Haynes and S. T. Hedetniemi, Client-server and cost effective sets in graphs, AKCE International Journal of Graphs and Combinatorics 15(2017), 211-2018.

[2] T.W. Haynes, S.M. Hedetniemi, S.T. Hedetniemi, T.L. McCoy, I. Vasylieva, Cost effective domination in graphs, Cong. Numer. 211 (2012), 197-209.

[3] S.M. Hedetniemi, S.T. Hedetniemi, A.A. McRae, Very cost effective bipartitions in graphs. AKCE International Journal of Graphs and Combinatorics. 12(2015), 155-160.

[4] F. Jamil and H. Maglanque, On cost effective domination in join, corona and composition of graphs, European Journal of Pure and Applied Mathematics, Graph theory. Vol.12, No.3, 978-998, 2019. 
[5] J. Palco, R. Paluga and G. Malacas, On k-cost effective domination number, cost effective domination index and maximal cost effective domination number of simple graphs, Far East Journal of Mathematical Sciences (FJMS), Volume 114, Issue 1, 55-68, 2019. 